LAWRENCE LIVERMORE NAT IO N A L LABORATORY

\section{A Final Focus Model for Heavy Ion Fusion Driver System Codes}

J. J. Barnard, R. O. Bangerter, E. Henestroza, I. D.

Kaganovich, B. G. Logan, W. R. Meier, D. V. Rose, P. Santhanam, W. M. Sharp, D. R. Welch, S. S. Yu

December 15, 2004

Nuclear Instruments and Methods in Physics Research A 
This document was prepared as an account of work sponsored by an agency of the United States Government. Neither the United States Government nor the University of California nor any of their employees, makes any warranty, express or implied, or assumes any legal liability or responsibility for the accuracy, completeness, or usefulness of any information, apparatus, product, or process disclosed, or represents that its use would not infringe privately owned rights. Reference herein to any specific commercial product, process, or service by trade name, trademark, manufacturer, or otherwise, does not necessarily constitute or imply its endorsement, recommendation, or favoring by the United States Government or the University of California. The views and opinions of authors expressed herein do not necessarily state or reflect those of the United States Government or the University of California, and shall not be used for advertising or product endorsement purposes. 


\section{A FINAL FOCUS MODEL FOR HEAVY ION FUSION}

\section{DRIVER SYSTEM CODES}

John J. Barnard ${ }^{1}$, Roger O. Bangerter ${ }^{2}$, Enrique Henestroza ${ }^{2}$, Igor D. Kaganovich ${ }^{3}$, B.

Grant Logan ${ }^{2}$, Wayne R. Meier ${ }^{1}$, David V. Rose ${ }^{4}$, Parthiban Santhanam ${ }^{2}$, William M.

Sharp ${ }^{1}$, Dale R. Welch ${ }^{4}$, Simon S. Yu ${ }^{2}$

1. Lawrence Livermore National Laboratory, Livermore, CA 94550

2. Lawrence Berkeley National Laboratory, Livermore, CA 94720

3. Princeton Plasma Physics Laboratory, Princeton, NJ 08543

4. Mission Research Corporation, Albuquerque, NM 87110

Corresponding author:

John J. Barnard

L-645

Lawrence Livermore National Laboratory

Livermore, CA, 94550 USA

Tel: $\quad$ 510-486-6124

Fax: $\quad 510-486-5392$

E-mail: $\quad$ jjbarnard@1lnl.gov

Keywords: inertial fusion energy, heavy-ion fusion, space-charge, emittance growth, final focus, beam neutralization, chromatic aberrations, geometric aberrations

PACS codes and keywords: 52.75.Di Accelerators and propulsion; 28.52.Av Fusion Reactors, Theory, Design and simulation

*This work performed under the auspices of the U.S Department of Energy by University of California, Lawrence Livermore and Lawrence Berkeley National Laboratories under contracts No. W-7405-Eng-48 and DE-AC03-76SF00098 and contract DEFG0295ER40919 at PPPL. 


\section{A FINAL FOCUS MODEL FOR HEAVY ION FUSION DRIVER SYSTEM CODES ${ }^{\dagger}$}

John J. Barnard ${ }^{1}$, Roger O. Bangerter ${ }^{2}$, Enrique Henestroza ${ }^{2}$, Igor D. Kaganovich ${ }^{3}$, B. Grant Logan ${ }^{2}$, Wayne R. Meier ${ }^{1}$, David V. Rose ${ }^{4}$, Parthiban Santhanam ${ }^{2}$, William M. Sharp ${ }^{1}$, Dale R. Welch ${ }^{4}$, Simon S. Yu ${ }^{2}$

1. Lawrence Livermore National Laboratory, Livermore, CA 94550 2. Lawrence Berkeley National Laboratory, Livermore, CA 94720 3. Princeton Plasma Physics Laboratory, Princeton, NJ 08543 4. Mission Research Corporation, Albuquerque, NM 87110

\begin{abstract}
The need to reach high temperatures in an inertial fusion energy (IFE) target (or a target for the study of High Energy Density Physics, HEDP) requires the ability to focus ion beams down to a small spot. System models indicate that within the accelerator, the beam radius will be of order centimeters, whereas at the final focal spot on the target, a beam radius of order millimeters is required, so radial compression factors of order ten are required. The IFE target gain (and hence the overall cost of electricity) and the HEDP target temperature are sensitive functions of the final spot radius on target. Because of this sensitivity, careful attention needs to be paid to the spot radius calculation. We review our current understanding of the elements that enter into a systems model (such as emittance growth from chromatic, geometric, and non-linear space charge forces) for the final focus based on a quadrupolar magnet system.
\end{abstract}

\section{INTRODUCTION}

The production of electric power from inertial fusion targets driven by heavy ion beams requires the ability to focus ion beams onto small targets. Systems models indicate that within the accelerator, the beam radius will be of order centimeters, whereas at the final focal spot on the target beam radii of order millimeters are required, so radial compression factors of order ten are required. Target gain, and hence the overall cost of electricity is a sensitive function of the final spot radius on target. In order to determine optimum beam, accelerator, target and other machine parameters a systems code IBEAM [1] has been developed, which uses algebraic relations to model many aspects of the driver system and to develop a self-consistent model of a heavy ion driven inertial fusion power plant. Because of the sensitivity of spot radius on target gain, careful attention needs to be paid to the part of the model that calculates the spot radius. For use as part of a previous systems assessment study [2], a model was created which estimated the contribution to the focal spot from various physical effects in the final focusing section of a driver. Since the time that model was published, numerical simulations, bench-marked by experiments, have lead to a greater

\footnotetext{
$\dagger *$ This work performed under the auspices of the U.S Department of Energy by University of California, Lawrence Livermore and Lawrence Berkeley National Laboratories under contracts No. W-7405-Eng-48 and DE-AC03-76SF00098 and contract DEFG0295ER40919 at PPPL.
} 
understanding of the processes that affect the final spot size. One of the major recent developments, has been a more quantitative understanding of neutralized ballistic transport through a final focusing chamber. Neutralized ballistic focusing experiments (the Scaled Final Focus Experiment [3], Neutralized Transport Experiment [4]), analytic calculations, simulations using the hybrid-PIC code LSP, all point to the feasibility of focusing highly neutralized $(>99 \%)$ beams. It has been found, however, that non-linear residual electric fields can cause emittance growth, and this has significant consequences for an optimized accelerator. In this paper, we update the focusing model, based on our most recent analytical and numerical calculations.

\section{ELEMENTS OF THE MODEL FOR THE FINAL SPOT RADIUS}

The beam edge: Our goal is to be able to estimate the spot radius over a wide range of accelerator configurations. For that reason and because the power requirement on target relies primarily on a knowledge of the lowest order moments, we confine ourselves to the simplest "envelope" description of the beam. The "envelope" should be regarded as the beam edge for transport within the accelerator, where the beam will likely be space charge dominated. For a uniform distribution of charge (an appropriate representation for a space charge dominated beam) the beam edges are designated $a, b$ (in the $x, y$ directions, respectively, transverse to the primary beam motion). For uniform distributions, the quantities $a$ and $b$ are twice the root mean square of the average of the respective transverse coordinates, i.e. $a=2<x^{2}>^{1 / 2}$ and $b=2<y^{2}>^{1 / 2}$. Since $a$ is not necessarily equal to $b$, we allow for elliptical beams (as required by some target models), which is a generalization to the model of ref. [2] which considered only circular beams. The envelope equations remain valid for non-uniform distributions (as long as elliptical symmetry is maintained, and the eccentricity of the elliptical density contours do not vary with radius [4], although the ellipticity may vary, with longitudinal position $z$.) For non-uniform distributions, $a$ and $b$ continue to represent twice the rms averages of the particle coordinates $x$ and $y$. At the target, the beam is expected to be emittance dominated, and because propagation from final focus to target tends to rotate the $\left(x, p_{\mathrm{x}}\right)$ and $\left(y, p_{\mathrm{y}}\right)$ phase space distribution (where $p_{\mathrm{X}}$ and $p_{\mathrm{y}}$ are the transverse momenta), the density distribution $n(x, y)$ on target will ideally be Gaussian with respect to $x$ and $y$. That is, $n(x, y)=n_{0} \exp \left[-2\left(x^{2} / a^{2}+\right.\right.$ $\left.\left.y^{2} / b^{2}\right)\right]$, where $\mathrm{n}_{0}$ is the density on-axis. For this distribution, the fraction (1$1 / \mathrm{e}^{2}$ ), i.e. approximately $86.4 \%$, of the beam is contained within the ellipse with semi-axes $a$ and $b$. Non-linear optics and non-linear space charge can change this value, but simulations indicate that Gaussian distributions at the target can be good approximations.

Envelope considerations: The rms envelope equations couple $a$ and $b$ through space charge:

$$
\begin{aligned}
& \frac{d^{2} a}{d z^{2}}=K a+\frac{2 Q}{a+b}+\frac{\square}{a^{3}}, \\
& \frac{d^{2} b}{d z^{2}}=\square K b+\frac{2 Q}{a+b}+\frac{\square,}{b^{3}} .
\end{aligned}
$$

Here $z$ is the longitudinal coordinate in the lab frame, $Q$ is the effective 
perveance, $\square_{\mathrm{x}}$ and $\square_{\mathrm{y}}$ are the unnormalized emittances in the $x$ and $y$ directions, and $K=B^{\prime}(z) /[B \square]$ is the magnetic quadrupole focusing function where $B^{\prime}$ is the magnetic gradient and $[B \square]$ is the particle rigidity. This equation is valid when the beam density distribution is elliptical (and characterized by a single ellipticity at each $z$ ), and when image forces and nonlinear forces can be neglected. In general, the emittances will vary with $z$ and an auxiliary equation would be needed to integrate eqs. (1) and (2), but for uniform density, mono-energetic beams the emittances are constant and the equations can be integrated.

Consider a region over which $K$ is constant (e.g. in a "hard" edged quad, or within the chamber where $K$ is zero. Multiplying eq. (1) by $a$ and eq. (2) by $b$, adding the result and integrating allows the calculation of an energy-like constant $\mathrm{H}$ given by:

$2 H=a \unlhd+b \square \square K a^{2}+K b^{2} \square 4 Q \ln (a+b) \square \frac{L_{k}^{2}}{a^{2}} \square \frac{\square t}{b^{2}}$

Here $a \square=d a / d z$ and $b \square=d b / d z$. After exit from the final focus magnet the beam will be converging towards the target with the following approximate conditions:

$K=0, a^{\prime}=-\square_{\mathrm{x}}, b^{\prime}=-\square_{\mathrm{y}}, a=\square_{\mathrm{x}} d$, and $b=\square_{\mathrm{y}} d$, where $d$ is the distance between the end of the last magnet and the focal spot, and $\square_{\mathrm{x}}$ and $\square_{\mathrm{y}}$ are the focusing half-angles. At the focal spot itself with radii $r_{\mathrm{X}}=a$ and $r_{\mathrm{y}}=b$, the smallest spot obtainable will be at a waist, in which case $a^{\prime}=b^{\prime}$ $=0$. (If either $a^{\prime}$ or $b^{\prime}$ are finite at the point of minimum area, we assume nevertheless that $a^{\prime}<<\square_{\mathrm{x}}$ and $b^{\prime}<<\square_{\mathrm{y}}$ ). We then find: $\square_{x}^{2}+\square_{y}^{2} \square \frac{\square_{x}^{2}}{r_{x}^{2}}+\frac{\square_{y}^{2}}{r_{y}^{2}}+4 Q \ln \frac{\square d\left(\square_{x}+\square_{y}\right) \square}{=r_{x}+r_{y}}$ 目

When $\square_{\mathrm{x}}$ and $\square_{\mathrm{y}}=0$, then eqs. (1) and (2) imply $a^{\prime \prime}-b^{\prime \prime}=0, a^{\prime}-b^{\prime}=\mathrm{const}=-\left(\square_{\mathrm{x}}-\square_{\mathrm{y}}\right)$, and $a-b=-\left(\square_{\mathrm{x}}-\square_{\mathrm{y}}\right) z+a_{0}-b_{0}$. If there is a true waist at the target $\left(a^{\prime}-b^{\prime}=0\right)$ then $=\square_{\mathrm{x}}-\square_{\mathrm{y}}$ $=0$, and by symmetry

$\square_{x}^{2}=\square_{y}^{2} \square 2 Q \ln \sqrt[\square d\left(\square_{x}+\square_{y}\right)]{r_{x}+r_{y}}=$. In the other extreme, $(Q=0)$, then eqs. (1) and (2) are separable with the well known approximate solutions:

$\square_{x}^{2} \square \frac{\square_{x}^{2}}{r_{x}^{2}}$ and $\square_{y}^{2} \square \frac{\square_{y}^{2}}{r_{y}^{2}}$. We thus infer that an approximate solution for arbitrary $Q$, $\square_{x}$, and $\square$, is given by:

$$
\begin{aligned}
& \square_{x}^{2} \square \frac{\square_{x}^{2}}{r_{x}^{2}}+2 Q \ln \frac{\square d\left(\square_{x}+\square_{y}\right)}{r_{x}+r_{y}} \square \text { and } \\
& \square_{y}^{2} \square \frac{\square_{y}^{2}}{r_{y}^{2}}+2 Q \ln \frac{\square d\left(\square_{x}+\square_{y}\right)}{r_{x}+r_{y}}
\end{aligned}
$$

Eq. (5) is thus only approximately true for intermediate values of $Q, \square_{x}$, and $\square_{y}$. However the sum of $\square_{\mathrm{x}}^{2}+\square_{\mathrm{y}}^{2}$ found from eq. (5) is consistent with the more exact relation eq. (4), so for the purposes of this paper we adopt eq. (5) as our generalization of the relation between the focusing angles, the final elliptical spot radii, the perveance and emittances for elliptical spots, but bearing in mind its approximate nature for intermediate values. Also, Eq. (5) is valid when $Q$, $\square_{x}$, and $\square_{y}$ are constants. When they are not constant eq. (5) still may be employed to approximately relate the final spot radius to the focusing angles $\square_{\mathrm{x}}$ and $\square_{\mathrm{y}}$ if the parameters are chosen appropriately. Because of the steep power of $r_{\mathrm{x}}$ and $r_{\mathrm{y}}$ in the emittance terms in eq. (3), the final emittance 
should be used in eq. (5). However, because of the logarithmic dependence of the radius in the perveance term, an appropriately averaged perveance through the fusion chamber is required.

\section{Contributions to the final emittance:}

To calculate the final normalized emittance at the target, contributions to emittance dilution throughout the machine must be included. In the systems model, an estimate of the normalized emittance of each beam as it leaves the injector is first obtained; to that are added contributions from: nonlinear errors in the magnetic fields of the focusing quadrupoles, chromatic aberrations and geometric aberrations in the final focus system, and the imperfect non-linear neutralization of the beam in the chamber. We expect these contributions to be uncorrelated and so the contributions are added in quadrature. Pointing errors are added separately in quadrature with the beam radius.

Injector and accelerator: The normalized emittance from the injector

Thxi is given by

$\square_{n x i}=\square_{n y i}=2\left(k T / m c^{2}\right)^{1 / 2} r_{i n j}$

Here $T$ is the temperature of the source (about $1000^{\circ} \mathrm{C}$ or $\sim 0.1 \mathrm{eV}$ for hot plate sources or $\sim 1 \mathrm{eV}$ for plasma sources), $m$ is the ion mass, $r_{\mathrm{inj}}$ is the source radius (determined by using the ChildLangmuir law to calculate current, under the constraint of voltage breakdown relations.) It has been found empirically (ref. [7]) that imperfect beam optics increases this ideal by about a factor of two, and so in the systems code this emittance was multiplied by two to take account of this effect.
In the accelerator, non-linear magnetic fields give rise to emittance growth (ref. [8]). Also, quadrupole strength errors can create small mismatches of the beam. If the energy from those mismatches is thermalized one may estimate the increase of emittance from each quadrupole and the result can be shown to have the same scaling as calculated in ref. [8]. The contribution to the emittance squared from each quadrupole is:

$$
\square\left(\square_{h x q}\right) \square 4 a^{2} \square^{2} Q \frac{\square}{=\frac{\square}{B_{q}}} \frac{B_{q}}{B^{2}}
$$

Here $\square B_{q}$ is the component of the error magnetic field at the beam radius, which varies randomly from magnet to magnet. Contributions from all quads are summed (again in quadrature) to obtain an estimate of the final normalized emittance $\square_{\mathrm{a}}$ at the end of the accelerator. Nominal values of $\square B_{q} / B_{q}$ of $0.1 \%$ are typically assumed in system code calculations. Note that $\square^{2} Q$ is proportional to the line charge density, which, in turn, is proportional to the potential energy across the beam. By adjusting the coefficient $\left(\square B_{q} / B_{q}\right)$, the sensitivity of spot size to emittance growth from a variety of mechanisms can be explored.

\section{Longitudinal emittance growth:As}

discussed in the next section, a spread in parallel momentum will lead to an increase in emittance as the beam passes through the final focusing system. Just as in the transverse case where nonlinearities give rise to transverse emittance growth, it is expected that voltage variations in the induction cell pulsers will give rise to momentum spread in the beam (ref.[9]). In this case, the normalized longitudinal emittance is proportional to the product of the 
momentum spread and the bunch length. At the $\mathrm{i}^{\text {th }}$ induction gap, an rms momentum spread $\left[p_{\mathrm{i}}\right.$ will be introduced as a result of the voltage error $\square V_{\mathrm{i}}$, such that $\square p_{\mathrm{i}}=(1 / 2)(p / V) \square V_{\mathrm{i}}$. (Here $p=(2 m q V)^{1 / 2}$ is the (non-relativistic) momentum, and $q V$ is the energy of an ion after it transits the $i^{\text {th }}$ gap.) It is assumed that the voltage errors ultimately are phase mixed and are not corrected soon after generation. Each gap thus contributes $\left[p_{\mathrm{i}} l_{\mathrm{i}}\right.$ to the longitudinal normalized emittance where $l_{\mathrm{i}}$ is the bunch length at the $i^{\text {th }}$ gap. The voltage increment at each gap is $\square V_{\mathrm{i}}=L d V / d s$, where $L$ is the distance between gaps, and $\mathrm{dV} / \mathrm{ds}$ is the average accelerating grradient. The minimum number of pulsers at each gap is $\square V_{\mathrm{i}} / V_{\text {pulser }}$, where $V_{\text {pulser }}$ is the maximum voltage standoff of each pulser, e.g. around $10 \mathrm{kV}$ for typical thyratrons. Assuming the errors from each pulser $\square V_{\text {pulser }}$ add stochastically, $\square V_{\mathrm{i}}=\left[(L d V / d s) V_{\text {pulser }}\right]^{1 / 2}$

( $\left.\square V_{\text {pulser }} / V_{\text {pulser }}\right)$. For purposes of obtaining estimates of momentum spread, values of $\square V_{\text {pulser }} / V_{\text {pulser }}$ of about one percent are deemed typical of standard present technology.

The momentum spread at the end of the accelerator $\square p_{\mathrm{a}}$ is thus given by:

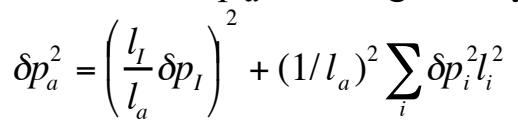

where $\square p_{\mathrm{I}}$ and $l_{\mathrm{I}}$ are the momentum spread and bunch length of the beam at the end of the injector, respectively, which is calculated separately from errors produced from the individual gaps. Incorporating the discussion preceding eq. (8) we find:

$$
\frac{\square p_{a}^{2}}{p_{a}^{2}}=\frac{1}{4 V_{a}^{2} \square t_{a}^{2}} \square\left[N_{I}^{2} \square t_{I}^{2}+\square \frac{\square N_{\text {pulser }}}{V_{\text {pulser }}}\right]^{2} \square V_{\text {pulser }} L \frac{d V}{d s} \square t_{i}^{2} \square
$$

Here $\square \mathrm{t}_{\mathrm{I}}, \square \mathrm{t}_{\mathrm{i}}$, and $\square \mathrm{t}_{\mathrm{a}}$ are the pulse durations at the injector, $i^{\text {th }}$ gap, and accelerator end, respectively, and $\square V_{I}$ is the voltage error on the injector, estimated to typically be of order $10^{-3}$ of the injector voltage. To estimate the momentum spread $\left[p_{\mathrm{f}}\right.$ at the final focus, one may assume conservation of longitudinal emittance, implying $\left[p_{\mathrm{f}} / \mathrm{p}_{\mathrm{f}}=\right.$ $C\left[p_{\mathrm{a}} / p_{\mathrm{a}}\right.$, where $C \equiv l_{\mathrm{a}} / l_{\mathrm{f}}$ is the compression ratio, and $p_{\mathrm{f}}$ and $l_{\mathrm{f}}$ are the ion momentum and bunch length at the final focus, respectively.

\section{Chromatic aberrations in final focus:}

In ref. [10], and ref. [11] theory and simulations suggested that the contribution to the normalized emittance from chromatic aberrations in the final focus magnets alone can be written in the form

$\square_{x c}=\square_{c x} d \frac{\mathrm{B} \square}{\square} \square \square_{x}^{2}$

Here $\square p$ is the rms longitudinal momentum deviation from the design momentum $p$. In ref. [11], $\square_{\mathrm{cx}}$ was an empirically derived constant found to be approximately 6 . We have derived a set of moment equations, which treats the fractional momentum spread $\square p / p$ as a first order quantity in the equations of motion. The set of moment equations, in effect, generalizes the envelope equations through second order. We briefly present the derivation of the moment equations in Appendix 1. Integration of the equations (A6), for particular focusing systems has found $\square_{c x}$ and $\square_{c y}$ in the range 4-12, although 
some systems with chromatic aberrations contributing anisotropically may have $\square_{\mathrm{cx}}$ as small as 2.4 , for example with $\square_{\text {cy }}$ approximately 6 . The integration (and hence determination of $\square_{c x}$ and $\left.\square_{\text {cy }}\right)$ depends sensitively on the phase of the matched envelope on which the initial value of $<x[p / p>$ is assumed to be zero. In principle, this evolves from a value of zero from the source, and is thus calculable for a given design; however for simulations of final focus for which only the last dozen or less lattice periods are included, the indeterminacy of $<x[p / p>$ leads to some uncertainty in the values of $\square_{c x}$ and $\square_{c y}$. Geometric aberrations in final focus: In ref. [12], an estimate was made of third order quantities for a heavy ion fusion focusing system. The result can be summarized in the following equation for the increase in the spot size from geometric aberrations

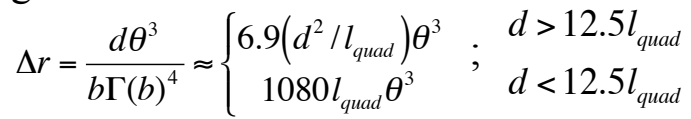

Here $\mathrm{d}$ is the final focal distance, $l_{\text {quad }}$ is the length of the final quadrupole magnet, and $b \equiv B_{\text {quad }} d /([B \square] \square) \square 1.25 d / l_{\text {quad }}$ where the latter approximate equality assumes: the thin lens approximation; the ratio of the aperture radius to the maximum radius of the beam envelope is 1.25; and the change in $a^{\prime}$ through the final lens is assumed to be equal to the convergence angle. Also, $\square[b]$ is a function defined in ref. [12] and approximated here in eq. (17).In ref. [12], $\square$ represented the initial emittance divided by the design spot radius, which for small aberrations is equal to the final focus convergence angle. Eq. (11) was based on a doublet final focus system (ref. [13]), a waist-towaist envelope trajectory, and space charge was absent in the calculation. Particle trajectories from linear fields were used as the unperturbed orbits and non-linearities from pseudo-octupole, and $\mathrm{B}_{\mathrm{z}}$, arising from the fringe fields of the quadrupoles and non-paraxial terms in the equations of motion were included. Simulations have found that, for four-quadrupole systems including space charge, the spot size given by eq. (11) overestimates the contributions of geometric aberrations by about a factor of3, for the parameters of the Neutralized Transport Experiment [4]. We have not yet analytically reevaluated the geometric aberrations in the context of a 4 or more quadrupole final focus system in the presence of space charge, analytically. We have parameterized our uncertainty in this quantity by introducing a factor $\square_{g}$, where the emittance growth from geometric aberrations $\bigsqcup_{\mathrm{gx}}$ is given by:

$\square_{\mathrm{gx}}=1080 \square \mathrm{g} l_{\text {quad }} \square_{\mathrm{x}}^{4}$

thus giving a spot radius $\square r$ from geometric aberrations alone of $\square r \sim$ $1080 \square \mathrm{g} l_{\text {quad }} \square_{\mathrm{x}}{ }^{3}$, which agrees with the scaling of eq. (11), but where the coefficient is numerically determined for a particular magnet layout, nominally assumed to be 0.32 .

\section{Emittance growth and neutralization} in the chamber: The topic of emittance growth and neutralization in the chamber has been the subject of comprehensive numerical and analytic study (refs. [15] through [28]). For simplicity, we have assumed ballistic neutralized transport. Plasma plug: Simulations have shown that when a "plasma plug" (a region of 
ionized chamber gas in electrical contact with the walls of the chamber) is available after the beam leaves the final focus magnet, the beam space charge will draw and entrain electrons into the beam providing nearly complete neutralization of the beam space charge as it propagates to the target. However, the neutralization is not perfect and this process will only continue until the beam potential is reduced to of order $\mathrm{m}_{\mathrm{e}} \mathrm{v}_{0}^{2} / 2$ (refs. [22-24]), where $m_{e}$ is the electron mass. This is because in the beam frame, the electrons will have this kinetic energy relative to the beam ions, and they will not be trapped or entrained by potentials that are lower than this energy. It is straightforward to show that the resulting beam perveance $Q_{c 0}$ (in the limit of large initial beam current) is given by:

$Q_{c 0} \square \square Q_{Q} Z_{e f f} m_{e} / m_{i}$

Here $\square_{Q}$ is a parameter of order unity (obtained from simulations), $Z_{\mathrm{eff}}$ is the effective charge state of the beam ions as they traverse the chamber (and due to stripping may not be equal to the initial charge state), and $m_{\mathrm{i}}$ is the ion mass. At very low ion currents, the perveance of the beam itself is less than given by eq. (13) and simulations (ref. [21]) show that for initial beam perveance less than $Q_{c 0}$, neutralization is ineffective, and for initial beam perveance greater than $Q_{c 0}$, the neutralized perveance approaches $Q_{c o}$, so that in general, the neutralized plasma plug perveance is given by:

$Q_{c} \square Q_{c 0}\left(1 \square \exp \left(\square Q_{b} /\left|Q_{c 0}\right|\right)\right)$

Here $Q_{\mathrm{b}}$ is the unneutralized beam perveance. Although the electrons provide overall charge neutralization, the distribution of electrons will not match perfectly the distribution of ions. The space charge field of the beam is therefore non-linear and the beam emittance grows as a result. Analytic work on beams with non-uniform space charge distributions [28] indicates that the emittance squared grows linearly with propagation distance according to:

$\frac{d\left(\bar{L}_{x}^{2}\right)}{d z^{2}} \square \frac{\square_{s c}^{2}}{2} Q^{2}$

Here $\square_{\text {sc }}$ (corresponding to $\square$ of ref. [28]), is a parameter that depends on the distribution of charge (of order $1 / 2$ for a variety of non-uniform charge distributions), and $\mathrm{Q}$ is the effective beam perveance. For propagation through the chamber, the contribution to the total emittance growth from nonlinear space charge is thus given by:

$\square_{b c} \square \frac{\square_{s c}^{2}}{4} Q_{c}^{2} d^{2}+\square \hat{b}$

Volumetric plasma neutralization:

When the target is heated by a "foot" pulse, X-rays will be emitted and can photoionize the chamber gas in the vicinity of the target. This will provide additional electrons to neutralize the beam, which can be supplied continuously along the ion beam path. Since this neutralization method does not require the electrons to become entrained with the ions, much higher space charge neutralization fractions than are given by eq. (13) and (14) can be obtained. In fact, the residual space charge potential may be regarded as negligible compared with the selffocusing effect of the residual current. In ref. [25], a theory of the residual net current of a beam propagating through a plasma has been obtained. It is based on finding solutions of the equations of motion of an electron fluid and Maxwell's equations. It was shown that Maxwell's equations and the equations of motion of the electrons fluid have a 
conservation law for the generalized vorticity $\square$ where $\square$ is defined as: $\square \equiv \square \square \mathbf{p}_{e} \square e \mathbf{B}$.

here $\mathbf{p}_{\mathrm{e}}$ is the electron fluid momentum and $\mathbf{B}$ is the self-magnetic field of the electron fluid and beam. In the limit of a long beam, where steady conditions arise, Ampere's law implies $\square \square \mathbf{B}=\square_{0} \mathbf{J}$ so that $\square\left(\square \square \mathbf{p}_{e}\right)=\square_{0} e \mathbf{J}$. For a cylindrical symmetrical beam, Stokes theorem implies $2 \square r\left(\square \square \mathbf{p}_{e}\right)_{z}=\square_{0} e I(r)$, where $I(r)$ is the longitudinal current within radius $r$. The total net current having a radial extent the radius of the beam $r_{\mathrm{b}}$ is thus given by:

$$
I_{n e t}=\frac{2 \square}{\square_{0} e}\left[r\left(\square \square \mathbf{p}_{e}\right)_{z}\right]_{r=r_{b}} \square \square_{m} \frac{2 \llbracket p_{e}}{\square_{0} e} \frac{r_{b}}{\square 2 r_{s}} \text { 目 }
$$

The final approximate equality in eq. (18) assumes the scale length for the change in $p_{\mathrm{e}}$ from center to beam edge is of order $r_{\mathrm{S}}$, and a coefficient $\square_{\mathrm{m}}$ of order unity is introduced to further reflect that it is an approximation. $\left[r_{s}\right.$ is approximately the minimum of the "skin depth" $\square_{\mathrm{p}}=c /\left(e^{2} n_{\mathrm{p}} /\left[m_{\mathrm{e}}\right)^{1 / 2}\right.$ and the beam radius $r_{\mathrm{b}}$.] At lowest order there is charge neutralization $\left(Z_{b} n_{b}+n_{p} \square n_{e}\right)$

(where $n_{\mathrm{b}}, n_{\mathrm{e}}$, and $n_{\mathrm{p}}$ are the beam, electron, and plasma iondensities respectively) and current neutralization $\left(q n_{b} v_{b} \square n_{e} v_{e}\right)$, so to lowest order $v_{e} \square v_{b}\left(Z_{b} n_{b} /\left(n_{p}+Z n_{b}\right)\right.$. This reduces to the limits $v_{e} \square v_{b}\left(Z n_{b} / n_{p}\right)\left(\right.$ if $\left.n_{\mathrm{b}}<<n_{\mathrm{p}}\right)$ or $v_{e} \square v_{b}$ (if $n_{\mathrm{b}}>>n_{\mathrm{p}}$ ).

The net current is thus approximately given by:

$$
I_{n e t} \square\left\lfloor Z_{b} n_{b} /\left(Z_{b} n_{b}+n_{p}\right)\right\rfloor\left(r_{b} / r_{s}\right) 2 \square\left[\square \left[n_{e} v_{b} c^{2} / e\right.\right.
$$

The contribution to the perveance from the net current $Q_{\mathrm{m}}$ is thus:

$$
Q_{m}=\frac{\square q e I_{n e t}}{2 \square \square m_{i} c^{3} \square_{b}}=\frac{\square \square_{m}}{1+f_{p}} \frac{q m_{e}}{m_{i}}=\frac{r_{b}}{\square 2 r_{s}} \text { 回 }
$$

Here $f_{p} \equiv n_{p} /\left(Z_{b} n_{b}\right)$. Note that the perveance is negative, resulting in a net focusing of the beam, but is less than (but can approach the order of assuming $\square_{\mathrm{sc}}=\square_{\mathrm{m}}, r_{\mathrm{b}}<\sim r_{\mathrm{s}}$ and $f_{\mathrm{p}>\sim 1)}$ the defocusing perveance from space charge in the case of a plasma plug (where electrons are entrained into the beam over a finite distance, in contrast to the present assumption of nearly unlimited electrons allowed to be dragged transversely into the beam over the entire length of propagation of the beam). (See ref. [25] for a complete and more rigorous derivation of eq. (20). ) This model may be appropriate for the steady state portion of the main pulse, for which a "foot" pulse has heated the target and preionized the plasma in the vicinity of the target, and a plasma "plug" has provided a plasma near the entrance of the chamber. Thus, eq. (20) may be used to estimate the final perveance in eq. (5), for the main pulse of the target. Note that the scaling for volumetric plasmas has been found to be quite different than plasma plugs (ref. [27]). In ref. [27] it is shown that the effective perveance is quite independent of initial beam perveance (in contrast to eq. (14)) and that the neutralization is most dependent on whether the quantity $\square_{\mathrm{p}} \square \mathrm{t}$ is $<<$ or $>>1$, the latter condition, required for validity of eq. (20). Here $\square_{p}$ is the electron plasma frequency and $\square \mathrm{t}$ is the ion beam pulse duration.

For determining emittance growth in the chamber, it is still appropriate to use eqs. (14) and (16), since the "plasma plug" perveance will determine the emittance growth for a large fraction of the chamber. 
In applying these equations, one should bear in mind that these are approximate relations, intended to obtain the correct scaling over variations in accelerator or beam parameters. At this point of our understanding, detailed simulations are still needed to accurately predict the spot radius for a given set of beam and plasma parameters.

\section{SUMMARY OF MODEL}

For an approximate determination of the semi major axes of the final elliptical spot in a systems code (such as IBEAM), a knowledge of the beam emittance and fractional momentum spread on entrance to the final focus section, perveance while propagating through the chamber, final convergence angle $\square$, and final focal distance $d$, are needed. The final emittance may be calculated by adding in quadrature estimates of the emittance from the various sources:

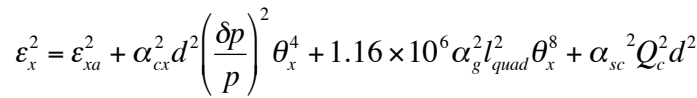

and

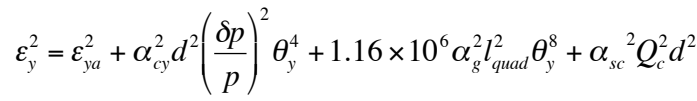

The approximate solutions to the envelope equations (eq. 5) can then be used to estimate the elliptical spot radii.

Example: 4.4. GeV, 1.1 kA Bismuth

beam: To demonstrate the magnitude of these various contributions to the focal spot we take as a "typical" example a beam from a driver with ion species Bismuth (209 amu), a single beam current of $1.9 \mathrm{kA}$, and ion energy 4.0 $\mathrm{GeV}$. (These parameters are similar to those for a main pulse of the "Robust Point Design" of ref. [30]). The $1.9 \mathrm{kA}$ is a result of a factor of seventeen increase in current in the drift compression section. For a source with radius $5 \mathrm{~cm}$ and temperature $1.0 \mathrm{eV}$ the normalized emittance of such a beam would be $0.23 \mathrm{~mm}$-mrad, which, allowing for optics imperfections, may be $\sim 0.46 \mathrm{~mm}$-mrad at the end of a 15 $\mathrm{cm}, 600 \mathrm{kV}$ injector diode. The current of such a beam would be $0.6 \mathrm{~A}$, through the end of a $2 \mathrm{MV}([=.00453)$ injector.

Accelerating, in a magnetic quadrupole lattice from $2 \mathrm{MeV}$ to $4 \mathrm{GeV}$, the line charge density increases by about a factor of 2.5 , so the quantity $\sim 4 \square^{2} Q a^{2}$ $=2 q \square a^{2} /\left(\square \square 0 m c^{2}\right)$ increases by this amount also. For a $2 \mathrm{~cm}$ beam, at 0.44 $\square \mathrm{C} / \mathrm{m}$, and $0.1 \% \square B_{\mathrm{q}} / B_{\mathrm{q}}$ the normalized emittance growth per quadrupole would be $8 \times 10^{-3} \mathrm{~mm}-\mathrm{mrad}$. Taking into consideration $\mathrm{d} \ln \square / \mathrm{d} \ln \mathrm{V}=0.12$, with an accelerator with $\sim 675$ quads, the normalized emittance has increased by only $0.33 \mathrm{~mm}$-mrad, which gives a combined emittance from injector and quads to be $0.57 \mathrm{~mm}$-mrad. This is somewhat ideal, but sets goals for what is possible. Similarly, the fractional parallel momentum spread can be estimated from eq. (9). For an initial pulse of $30 \square \mathrm{s}$ that is compressed to 170 $\mathrm{ns}$ in the accelerator and then down to 10 ns at the final focus, using $\mathrm{d} \ln \square t / \mathrm{d} \ln V=$ 0.62 , we find a contribution to $\left[p / p_{\mathrm{f}}\right.$ of 6 $\mathrm{x} 10^{-4}$, from errors in the induction gaps. For an injector voltage error of $10^{-3}$, we find a contribution to $\square p / p_{\mathrm{f}}$ of $4.5 \times 10^{-4}$, so that the predicted total $\square p / p_{\mathrm{f}}$ is $7.5 \mathrm{x}$ $10^{-4}$.

With this fractional momentum spread, a final focus distance of $6 \mathrm{~m}$, a final focusing angle $\square$ of $10 \mathrm{mrad}$, and $\square_{\mathrm{cx}}$ of 4, then hex is $0.35 \mathrm{~mm}$-mrad. With these parameters, and $\square_{\mathrm{g}}=0.32$, a $1.6 \mathrm{~m}$ final magnet, then eq. (18) (emittance 
from geometric aberrations) suggests that $\square_{\mathrm{hg}}=2.0 \mathrm{~mm}-\mathrm{mrad}$. (Note that in calculating chromatic and particularly geometric aberrations, a magnet layout is generally needed. For ref. [30] the layout involved a number of constraints based on spot size, magnet construction difficulty, magnet replacement rate (minimization of magnet activation), and thresholds for angular array extent (dictated by target considerations) and maximum tolerable beam ellipticity (to avoid additional emittance growth).) Finally, while propagating in the chamber, for this example, from eq. (19) and (20), a final perveance of $5.2 \times 10^{-6}$, could be reached, if $\square \mathrm{Q} Z_{\text {eff }}=2$, and a "plasma plug" is the source of neutralization. This would lead to a normalized emittance contribution $\square_{\mathrm{hsc}}=$ $3.1 \mathrm{~mm}-\mathrm{mrad}$ (if $\square_{\mathrm{sc}}=0.5$ ). The sum of all normalized emittances is $3.7 \mathrm{~mm}$ mrad, in this example, which, when included with the perveance term in eq. (5), yields a final spot size of $r_{\mathrm{x}}$ of 2.2 $\mathrm{mm}$. If photoionization of chamber gas by the heated target, then the plasma density may be as high as $3.5 \times 10^{13} \mathrm{~cm}^{-}$ 3 , and the beam density may be as high as $7.1 \times 10^{12} \mathrm{~cm}^{-3}$, so that $f_{\mathrm{p}}$ may be of order unity and $r_{\mathrm{b}} / 2 r_{\mathrm{S}}$ may also be of order unity, so that the final perveance may be $\sim-5 \times 10^{-6}$. If the emittance growth is assumed to be the same (due to the same conditions as occur in the plasma plug throughout most of the chamber, but that the final perveance determines the contribution to the space charge term in eq. (5)) then the final spot radius would be $1.6 \mathrm{~mm}$. Both results would meet the target requirements. This example illustrates the most recent realization that neutralization in the chamber (and the associated non-linear electric fields) may be the dominant source of beam emittance throughout the entire system. When the equilibrium perveance is larger than the bare beam perveance, then the final perveance and emittance growth (and hence spot size) is largely independent of beam parameters. However, for small enough beams neutralization (and associated emittance growth) may not be significant and the other terms in eq. (27) may be the largest contributors.

\section{IMPROVEMENTS TO THE MODEL \\ Corrections: Corrections of beam} aberrations using non-linear optics have been suggested by several authors and have not been included in the focal spot model. These include corrections to geometric aberrations using octupoles [32], and corrections to chromatic aberrations (using combinations of sextupoles/dipoles) [33]. Timedependent focusing has also been suggested to correct chromatic aberrations (refs. [31], [34], and [35]). This latter idea is that at the beginning of the drift compression, there is a strong correlation between longitudinal position and velocity. One could then, in principle, give each velocity class upstream (before drift compression) a time-dependent variation in the quadrupole field strength such that when that velocity class passes through the final focus optics, each velocity class will focus to the correct spot, thus minimizing chromatic aberrations [31]. Two possible problems are 1) the correct quadrupole variations upstream to optimize the focal spot may cause the beam to be mismatched, and 2) space charge may cause various velocity classes to be mixed at the final focus so the correction may not be so effective, 
(but there could still be some benefit). Multiple corrections might also be made during the drift compression. Correcting closer to final focus reduces the precision needed, as there are fewer betatron periods between the correction point and the final focus, but the correlation between velocity and longitudinal position is going down, so the corrections may become less effective the closer the beam is to final focus. Also, as the pulse duration decreases towards $10 \mathrm{~ns}$, the technological challenges may increase. The efficacy of these various correction schemes has yet to be determined. Assuming that they are successful, their impact on the overall configuration of an optimized accelerator and on the cost of electricity can be determined by setting

$\square_{\mathrm{cx}}, \square_{\mathrm{cy}}, \square_{\mathrm{g}}$ to zero or to some small value.

Emittance growth model: In addition to voltage errors contributing to the longitudinal emittance, simulations show that a transverse/longitudinal instability will grow until the longitudinal temperature is roughly a third to half of the transverse temperature. For an initial transverse temperature $\mathrm{kT}$ of $2 \mathrm{eV}$, an

injection voltage $\mathrm{V}_{\mathrm{I}}$ of $2 \mathrm{MV}$, the corresponding $\square \mathrm{p} / \mathrm{p} \sim$

$(\mathrm{kT} /[4 \mathrm{qV} \mathrm{I}])^{1 / 2} \sim 5 \times 10^{-4}$, so it is apparent that injector voltage waveform errors are initially somewhat more important than thermalization of transverse temperature, but this should be tracked throughout the accelerator. Another source of longitudinal emittance growth is timing jitter from the confining ("ear") waveform pulsers. This will be a source of space charge waves that can propagate to the interior of the beam. Even if the waves do not thermalize they will contribute to chromatic aberrations to the focal spot if not corrected. A simple model to account for jitter could be incorporated into the emittance growth model.

\section{CONCLUSION}

We have constructed a source-to-target model of the emittance evolution of a heavy ion beam. We have used the envelope equations to estimate the semimajor axes of the beam spot radius on the target including the combined effects of: emittance contributions from the injector temperature, quadrupole focusing errors in the accelerator, chromatic and geometric effects in final focus, non-linear and incomplete neutralization in the chamber. The chromatic effects are determined from an estimate of longitudinal velocity spread arising from injector and induction gap voltage errors through the accelerator. We have estimated the size of the various effects for one example set of parameters, and have found that the contribution from non-linear space charge effects in the chamber can be the dominant source of emittance growth, and is insensitive to beam parameters. Further refinement of these estimates, using PIC codes for benchmarking is in progress.

\section{REFERENCES}

[1] W. Meier, R. Bangerter, A. Faltens, Nucl. Inst. and Meth. in Phys. Res. A, "An integrated systems model for heavy ion drivers," 415, 249 (1998). Also, W. Meier, R. Bangerter, J. Barnard, “A 3.3 $\mathrm{MJ} \mathrm{Rb}+1$ driver design based on an integrated systems analysis," Nucl. Inst. and Meth. in Phys. Res. A, 464, 433 (2001).

[2] Edward P. Lee, "Accelerator and Final Focus Model for an Induction Linac Based HIF System Study," AIP 
Conference Proceedings 152, Heavy Ion Inertial Fusion, Washington, D.C. 1986, editors, M.Reiser, T. Godlove, and R. Bangerter [American Institute of Physics, New York] p. 461 (1986). [3] S. MacClaren, A. Faltens, P. A. Seidel, "Results from a scaled final focus experiment,"Physics of Plasmas, 9, 1712-1720 (2002).

[4] P.K. Roy et al, "Results on intense beam focusing and neutralization from the neutralized beam experiment," Physics of Plasmas, 11, 2890 (2004). (See also, P.K. Roy et al, "Neutralized Transport Experiment," these proceedings.)

[5] D. A. Callahan-Miller and M. Tabak, "Progress in Target Physics and Design for Heavy Ion Fusion," Phys. of Plasmas, 7, 2083 (2000).

[6] F. J. Sacherer, "RMS envelope equations with space charge," IEEE Transactions on Nuclear Science, NS-18, 1105 (1971).

[7] J.W. Kwan, L. Ahle, D.N. Beck, F.M. Bieniosek, A. Faltens, D.P. Grote, E. Halaxa, E. Henestroza, W.B. Herrmannsfeldt, V. Karpenko, T.C. Sangster, "Ion sources and injectors for HIF induction linacs," Nuclear Instruments and Methods in Physics Research A, 464, 379, (2001). [8] E.P. Lee, HIFAR Note \#486 "Rough Estimate of Emittance Growth from Magnetic Field Errors," February 19, 1999 (1999).

[9]A. Warwick and E.P. Lee, "Accelerator errors in MBE-4 and a driver,"HIFAR note 214 (1988). [10] E.P. Lee, "Partial cancellation of the second order chromatic aberration of a quadrupole focusing system by space charge forces," LBL-28463 (abstract), APS DPP meeting, (1991). [11] D. A. Callahan, "Chamber propagation physics for heavy ion fusion," Fusion Engineering and Design, 32-33 441 (1996).

[12] D. Neuffer, "Geometric Aberrations in Final Focusing for Heavy Ion Fusion," Proceedings of the Heavy Ion Fusion Workshop Held at Argonne National Laboratory, Sept. 19-26, 1978, ANL-79-41, p. 333 (1978).

[13] A. A. Garren, "Final Focusing the Ion Beams of a Pellet Fusion Reactor by Quadrupole Doublets," ERDA Summer Study of Heavy Ions for Inertial Fusion, LBL-5543, July, 1976, Appendix 8-7, p. 102 (1976).

[14] D.D.-M. Ho, I. Haber, and K.R. Crandall, "Octupole Correction of Geometric Aberrations for High-Current Heavy Ion Beams," Particle Accelerators, 36, 141, (1991). [15]D.V. Rose, D.R.Welch, B.V. Oliver, R.E. Clark, W.M. Sharp, and A. Friedman, Nucl. Inst. and Meth. Phys. Res. A 464, 299 (2001). [16] B.V. Oliver, T. Genoni, D. Rose, and D. Welch, "Laminar equilibria of ion-beam neutralizing electrons in vacuum and plasma," Self-Pinch Workshop for Final Transport of Heavy Ion Beams, February 13, 2001 [17] D.R. Welch, D.V. Rose, B.V. Oliver, T.C. Genoni, R. E. Clark, C.L. Olson, and S.S. Yu, "Simulations of intense heavy ion beams propagating through a gaseous fusion target chamber," Phys. Plasmas, 9, 2344 (2002).

[18]W.M. Sharp, D.A. Callahan, M.Tabak, S.S. Yu, P.F. Peterson, D.R. Welch, D.V. Rose, and C.L. Olson, "Modeling Chamber Transport for Heavy Ion Fusion," Fusion Science and Technology (2002). [19]W.M. Sharp, D.A. Callahan Miller, M.Tabak, S.S. Yu, P.F. Peterson, "Chamber transport of "foot" pulses for 
heavy ion fusion", Physics of Plasmas 10, 2457 (2003).

[20] D. V. Rose, D.R. Welch, B. V.

Oliver, C.L.Olson, "Neutralization of intense ion beams by localized plasmas," MRC/ABQ-R-2025 (2001). [21] D.R. Welch, D.V. Rose, W.M. Sharp, C.L. Olson, S.S. Yu," Effects of preneutralization on heavy ion fusion chamber transport,"Lasers and Particle Beams, 20, 621, (2002)

[22] C. Olson, D. L. Hanson, J.W. Poukey, and D.R. Welch, "Microcharge neutralization transport experiments and simulations for ion-driver inertial confinement fusion," Fusion Engineering and Design, 32-33, 485 (1996).

[23] S.Humphreys, T.R. Lockner, J.W. Poukey, and J.P. Quintenz, Phys. Rev. Lett., 46, 995, (1981).

[24] R.N. Sudan, "Neutralization of a propagating intense ion beam in a vacuum," App. Phys. Letters, 44, 958, (1984).

[25] I. D. Kaganovich, E.A.Startsev, R.C. Davidson, "Analytical and Numerical Studies of Heavy Ion Beam Transport in the Fusion Chamber," Lasers and Particle Beams, 20, 497 (2002).

[26]. I. D. Kaganovich, G. Shvets, E. Startsev, R.C. Davidson, "Nonlinear charge and current neutralization of an ion beam pulse in a pre-formed plasma," Physics of Plasmas, 8, 4180 (2001). [27] I.D. Kaganovich, E. A. Startsev, R.C. Davidson,D.Welch,"Theory of Ion Beam Pulse Neutralization by a Background Plasma in a Solenoidal Magnetic Field," these proceedings. [28] E.P.Lee, S.S. Yu, W.A. Barletta, "Phase Space Distortion of a Heavy-Ion Beam propagating through a Vacuum Reactor Vessel," Nuclear Fusion, 21, 961 (1981).
[29] E. Henestroza, "NTX: A

Neutralized Final Focus System for High Intensity Beams," Physical Reveiew:Special Topics Accelerators and Beams , in press (2003). [30] S.S. Yu, W.R. Meier, R.P Abbott, J.J. Barnard, T. Brown, D.A. Callahan, C.S. Debonnel, P. Heitzenroeder, J.F. Latkowski, B.G. Logan, S.J. Pemberton, P.F. Peterson, D.V. Rose, G.-L. Sabbi, W.M Sharp, D.R. Welch, "An updated point design for heavy ion fusion," Fusion Science and Technology, 44 (2), 266-273, September 2003

[31] A. Maschke, (1976) (transmitted by R. Bangerter, private communication, 1996)

[32] D.D.-M. Ho, I. Haber, K.R.

Crandell, "Octupole Correction of

Geometric Aberrations for High-Current Heavy Ion Beams," Particle Acclerators, 36, 141 -160 (1991).

[33] D.D.-M. Ho, I. Haber, K.R.

Crandell, "Dependence of Quadrupole

Pole-Tip Fields on Beam Parameters and Design of Nondispersive Optics for final Focusing Systems for High Current Heavy Ion Beams LLNL Report UCRLJC-109998 (1992).

[34] H. Qin, R.C. Davidson, J.J.

Barnard, E.P. Lee, "Drift compression and Final Focus of Intense Heavy Ion Beams," Proceedings of the 2003

Particle Accelerator Conference, Portland, Oregon (2003).

[35] H. Qin, R.C. Davidson, J.J.

Barnard, E.P. Lee, "Drift compression and Final Focus Options for Heavy Ion Fusion," these proceedings (2004).

\section{Appendix I. Moment equations for chromatic aberrations}

We start with the equation of motion for a beam ion in a quadrupolar external field and include the effects of space 
charge distributed in an elliptical distribution. The $x$-component of the equation of motion is :

$\frac{d p_{x}}{d t}=q\left(E_{x}+v_{z} B_{y} \square v_{y} B_{z}\right)$

Here $E_{\mathrm{X}}$ is the $x$ component of the space charge field of the beam, and $B_{\mathrm{y}}$ and $B_{\mathrm{z}}$ are $y$ and $z$ components of the magnetic field of the quadrupole. Expanding through second order in $x^{\prime}, y^{\prime}, k_{\square 0} x$, $k_{\square} a$, $[p / p$ yields:

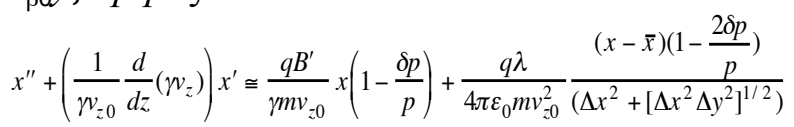

This equation (when $d\left(\square_{\mathrm{Z}}\right) / d z=0$ ) can be written:

$x \llbracket \square K_{x x} x+K_{x x 1} x \square p / p$.

Similarly, the y-equation of motion can be written

$y \llbracket \square K_{y y} y+K_{y y 1} y[p / p$.

Here, $K_{x x}= \pm \frac{B \square}{[B \square]_{0}}+\frac{2 Q}{2\left(\square x^{2}+\left[\square x^{2} \square y^{2}\right]^{1 / 2}\right)}$,

$$
\begin{aligned}
& K_{x x 1}=\square\left[\frac{B \square}{[B \square]_{0}}+\frac{2 Q}{2\left(\square x^{2}+\left[\square x^{2} \square y^{2}\right]^{1 / 2}\right)}\right] \text {, } \\
& K_{y y}=\frac{\mp B \square}{[B \square]_{0}}+\frac{Q}{2\left(\square y^{2}+\left[\square x^{2} \square y^{2}\right]^{1 / 2}\right)},
\end{aligned}
$$

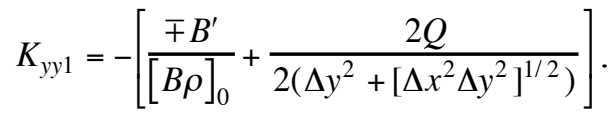

Here, $B \square=$ magnetic gradient, $[B \square]=$ ion

rigidity $=p / q ; Q=$ perveance

$=q \square /\left(2 \square \square \square \square_{0}^{3} m v_{z 0}^{2}\right)$.

Let $\square=\square p / p$. Noticing that

$\frac{1}{1+\square}=1 \square \square+\square^{2}+\ldots$ and

$\frac{1}{1 \square \square}=1+\square+\square^{2}+\ldots$ so that

$\frac{1}{1 \square \square} \square \frac{1}{1+\square}=2 \square+2 \square^{3}+\ldots$ and also

$\frac{\square}{1+\square}=1 \square \frac{1}{1+\square}$
We may perform averages over the distribution function and obtain the set of moment equations:

$$
\begin{aligned}
& \frac{d}{d s}\left\langle x^{2}\right\rangle=2\langle x x \mathrm{D}\rangle \\
& \frac{d}{d s}\left\langle x x \square=\left\langle x \square^{2}\right\rangle+K_{x x}\left\langle x^{2}\right\rangle+\frac{K_{x x 1}}{2}\left[\frac{x^{2}}{10 \square}\right\rangle\left\langle\left(\frac{x^{2}}{1+\square}\right) \stackrel{\square}{\square}+O\left(x^{2} \square^{3}\right)\right.\right.
\end{aligned}
$$

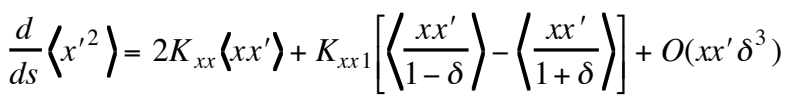

$$
\begin{aligned}
& \frac{d}{d s}\left\langle\frac{x x \square}{1+\square}\right\rangle=\left\langle\frac{x x^{\natural}}{1+\square}\right\rangle+K_{x x}\left\langle\frac{x^{2}}{1+\square}\right\rangle \square K_{x x 1}\left\langle\frac{x^{2}}{1+\square}\right\rangle+K_{x x 1}\left\langle x^{2}\right\rangle \\
& \frac{d}{d s}\left\langle\frac{x \square}{1+\square}\right\rangle=2 K_{x x}\left\langle\frac{x x \square}{1+\square}\right\rangle+2 K_{x x 1}\left\langle x x \square \square 2 K_{x x 1}\left\langle\frac{x x \square}{1+\square}\right\rangle\right.
\end{aligned}
$$

The equations for $y$ are identical with the substitution $y->x$, in all variables and subscripts. Note, that the error terms in eq. (A6) are two orders of $\square$ higher than the effective order of the term in brackets and are thus dropped. In practice, this limits the integration distance over which the equations may be used, but over a short number of lattice periods as in a final focus system, the system of equations is quite accurate. When candidate final focus systems are evaluated we find that the scaling $\square \mu \square p / p$ is satisfied, and when the factors $\square_{\mathrm{x}}, \square_{\mathrm{y}}$, and $d$ are included in the normalization of the relation, that the factors $\square_{\mathrm{cx}}$ and $\square_{\mathrm{cy}}$ depend on the particular final focus system (and on the initial phase of the matched period for which the value of $\langle x \square \nabla=0$ is assumed), but are in the range 4-12. 
Table 1: Summary of constants in emittance growth model:

\begin{tabular}{|l|l|l|}
\hline Quantity & $\begin{array}{l}\text { Nominal } \\
\text { Value }\end{array}$ & Comments \\
\hline$\square_{\mathrm{cx}}, \square_{\mathrm{cy}}$ & 4 & $\begin{array}{l}\text { normalized emittance growth from } \\
\text { chromatic aberrations; dependent } \\
\text { on final focus magnet layout, and } \\
\text { initial correlation of } x \text { and }[\mathrm{p} / p\end{array}$ \\
\hline$\square_{\mathrm{g}}$ & 0.32 & $\begin{array}{l}\text { normalized emittance growth from } \\
\text { geometric aberrations; dependent } \\
\text { on final focus magnet layout }\end{array}$ \\
\hline$\square_{\mathrm{Q}}$ & 2 & $\begin{array}{l}\text { Normalization constant in final } \\
\text { perveance for "plasma plug" } \\
\text { neutralization methods }\end{array}$ \\
\hline$\square_{\mathrm{m}}$ & 1 & $\begin{array}{l}\text { Normalization in final perveance } \\
\text { for "zero vorticity" model }\end{array}$ \\
\hline$\square_{\mathrm{sc}}$ & 0.5 & $\begin{array}{l}\text { normalization in emittance growth } \\
\text { rate due to space charge non- } \\
\text { linearity; depends on distribution } \\
\text { of space charge }\end{array}$ \\
\hline
\end{tabular}

Table 1: Summary of dimensionless parameters of order unity used in calculating the final ion beam emittance on the spot. Generally, simulations are required to determine each $\square$. 